\title{
Image Quality Assessment Using Spiking Neural Networks
}

\author{
Vijayalakshmi.C1,Joshua arul kumar.R2 \\ 1Student,M.E(ECE),ECE,MAMCE,Trichy,Tamilnadu,India, \\ 2AssociateProfessor,ECE,MAMCE,Trichy,Tamilnadu,India,
}

\begin{abstract}
An image quality assessment method was developed using spiking neural network and support vector machines (SVM) with the peak signal to noise ratio (PSNR) and root mean square error(RMSE) used to describe image quality. The neural network was used to obtain the mapping functions between the objective quality assessment indexes and subjective quality assessment. The SVM was used to classify the images into different types which were accessed using different mapping functions. The number of isolated points in the correlations of the image subjective and objective quality assessments was reduced by this method. Simulation results show that the method accurately accesses image quality.

Index Terms: image quality assessment; neural network; isolated points; PSNR;RMSE.
\end{abstract}

\section{Introduction}

Most images are compressed with losses before transmission. Therefore ,methods are needed to evaluate image quality with the reconstructed images quality directly reflecting the performance of the compression method, much attention has been placed on objective quality assessments which reflect the subjective image and video subjective quality with the main task being to reduce the offset between subjective and objective assessments. Images are assessed based on the human visual system (HVS), but the HVS is more complicated than the peak signal to noise ratio (PSNR) and some physiological and psychophysical characteristics of the HVS are not yet well understood. PSNR does not involve much characteristic information concerning the image, so distinct differences occur between the PSNR and the subjective image quality. The PSNR and SSIM are used as indexes describing the image quality.

\subsection{A Generic Spiking Neuron}

The elementary processing units in the brain are the neurons, which are connected to each other in an intricate pattern and can occur in many shapes and sizes. As stated above, the neuron described here is very simplified and its purpose is to serve as a 'template' neuron for further development.

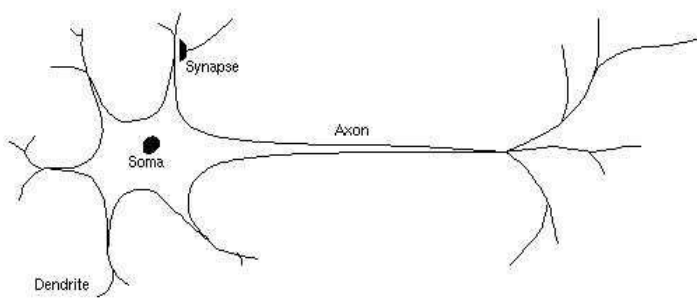

Figure 1.1: A structure of a spiking neuron

Figure1.1 gives the structure of a spiking neuron. Our generic neuron has four functionally distinct parts, called dendritic tree, soma, axon and synapse. Roughly speaking, signals from other neurons are collected by the dendrites (input device) and are transmitted to the soma (central processing unit). If the total excitation caused by the input is sufficient, i.e., above a threshold, an output signal (action potential, or spike) is emitted and propagated along the axon (output device) and its branches to other neurons. It is in the transition zone between the soma and the axon hillock, where the the essential non-linear processing step occurs.

\section{2 spiking neural networks}

Spiking neural networks (SNN) represent a special class of artificial neural networks (ANN), where neuron models communicate by sequences of spikes. Networks composed of spiking neurons are able to process substantial amount of data using a relatively small number of spikes.Due to their functional similarity to biological neurons, spiking models provide powerful tools for analysis of elementary processes in the brain, including neural information processing, plasticity and learning. At the same time spiking networks offer solutions to a broad range of specific problems in applied engineering, such as fast signal-processing, event detection, classification, speech recognition, spatial navigation or motor control. It has been demonstrated that 
SNN can be applied not only to all problems solvable by non-spiking neural networks, but that spiking models are in fact computationally more powerful than perceptrons and sigmoidal gates. Due to all these reasons SNN are the subject of constantly growing interest of scientists.

\subsection{Feedforward networks}

\section{Classification Of Snn}

This is where the data flow from input to output units is strictly one-directional; the data processing can extend over multiple layers of neurons, but no feedback connections are present. In biological neural systems feedforward projections can be found mainly in areas closer to the periphery. Similarly, in SNN feedforward topologies are usually app to model low-level sensory systems, e.g. in vision, olfaction or tactile sensing. Feedforward networks are investigated also in the context of spike synchronization or for solving the binding problem based on spatio-temporal patterns of spikes.

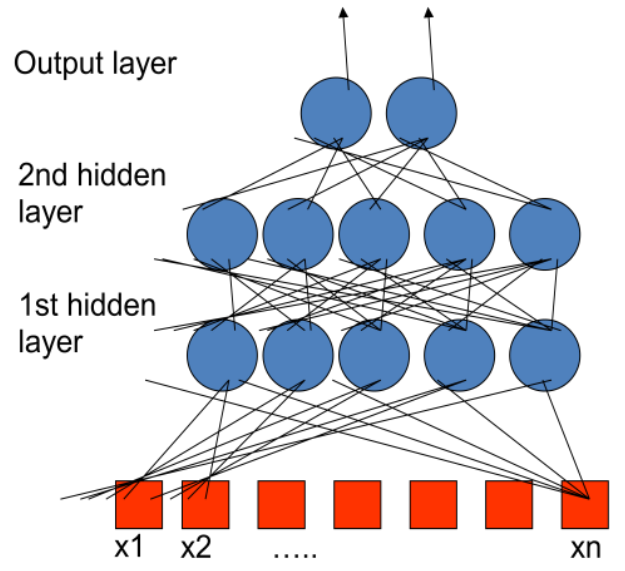

Figure 1.2:feedforward networks

\subsection{Recurrent networks}

Here individual neurons or populations of neurons interact through reciprocal (feedback) connections. Feedback connections result in an internal state of the network which allows it to exhibit dynamic temporal behavior. Consequently recurrent networks are characterized by richer dynamics and potentially higher computational capabilities than feedforward networks. Unfortunately, they are also more difficult to control and train (Hertz et al. 1991).

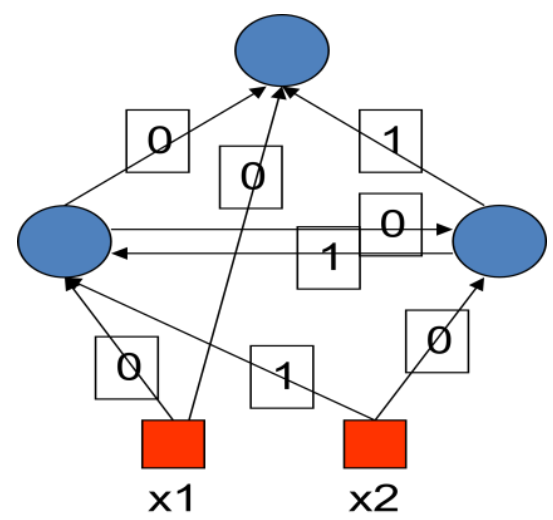

Figure 1.3: recurrent networks

\section{Learning Process:}

The procedure that consists in estimating the parameters of neurons so that the whole network can perform a specific task.It contains 2 types of learning namely,

1) The supervised learning

2) The unsupervised learning

The Learning process (supervised)

i. Present the network a number of inputs and their corresponding outputs

ii. See how closely the actual outputs match the desired ones 
iii. Modify the parameters to better approximate the desired outputs

\subsection{Supervised learning}

The desired response of the neural network in function of particular inputs is well known.A "Professor" may provide examples and teach the neural network how to fulfill a certain task.

\subsection{Unsupervised learning:}

$>$ Idea : group typical input data in function of resemblance criteria un-known a priori

$>$ Data clustering

$>$ No need of a professor

- The network finds itself the correlations between the data

- Examples of such networks :

$\checkmark$ Kohonen feature maps

\section{Spiking Neuron Models:}

Biological neurons communicate by generating and propagating electrical pulses called action potentials or spikes (du Bois-Reymond 1848, Schuetze 1983, Kandel et al. 1991). This feature of real neurons became a central paradigm of a theory of spiking neural models. From the conceptual point of view, all spiking models share the following common properties with their biological counterparts: (1) They process information coming from many inputs and produce single spiking output signals; (2) Their probability of firing (generating a spike) is increased by excitatory inputs and decreased by inhibitory inputs; (3) Their dynamics is characterized by at least one state variable; when the internal variables of the model reach a certain state, the model is supposed to generate one or more spikes. Historically the most common spiking neuron models are Integrateand-Fire (IF) and Leaky-Integrate-and-Fire (LIF) units. Both models treat biological neurons as point dynamical systems. Accordingly, the properties of biological neurons related to their spatial structure are neglected in the models. The dynamics of the LIF unit is described by the following formula:

$$
C \frac{d u}{d t}(t)=-\frac{1}{R} u(t)+\left(, \text { io }(\mathrm{t})+\sum \mathrm{wj}+\mathrm{ij}(\mathrm{t})\right)
$$

where $\mathrm{u}(\mathrm{t})$ is the model state variable (corresponding to the neural membrane potential), $\mathrm{C}$ is the membrane capacitance, $R$ is the input resistance, io(t) is the external current driving the neural state, $i j(t)$ is the input current from the $\mathrm{j}$-th synaptic input, and wj represents the strength of the $\mathrm{j}$-th synapse. For $\mathrm{R} \rightarrow \infty$, formula (1) describes the IF model. In both, IF and LIF models, a neuron is supposed to fire a spike at time tf , whenever the membrane potential $u$ reaches a certain value $v$ called a firing threshold.

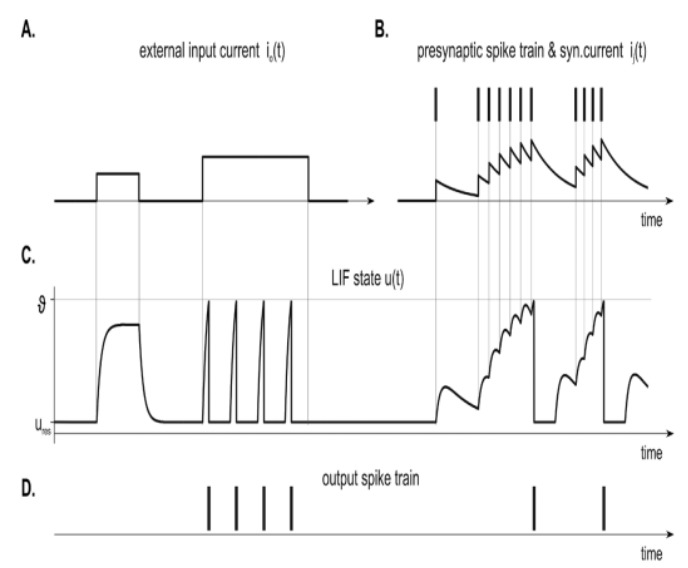

Figure 1.4: leaky-integrate-and-fire neuron LIF

which spiking activity can propagate as a synchronous wave of neuronal firing (a pulse packet) from one layer (subpopulation) of the chain to the successive ones (Diesmann et al. 1999). 


\section{Quality Assessments Using Spiking Neural Network And Svm}

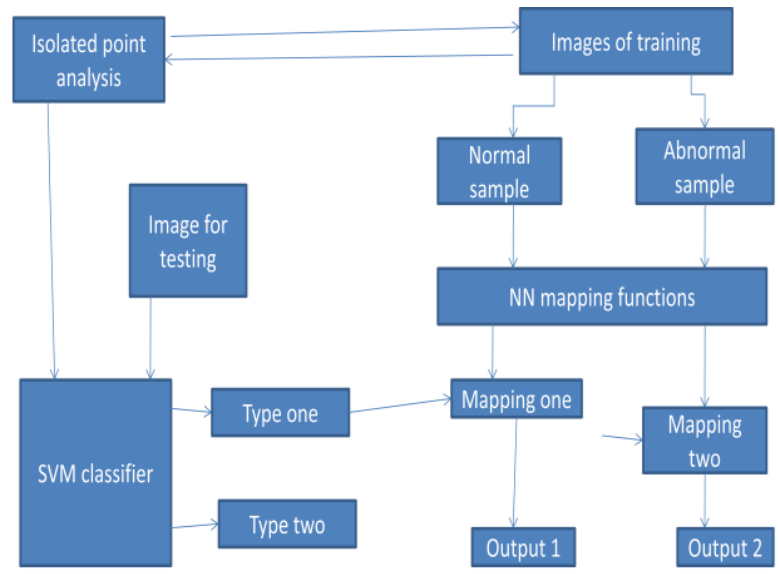

Figure 1.5:Image quality assessment

The neural network and SVM methods were combined to set up a new method with image quality assessment indexes ,such as PSNR,BIAS,MAD,RMSE,CC,ENTROPY DIFFERENCE,ERGAS AND RASE. The flow chart is shown in Figure 1.4. The image quality assessment is divided into training and testing parts.If the offset between the assessments is larger than a threshold, then the image corresponding to that point in the curve is known as isolated point. In the training part, the isolated points were analyzed with the mapping functions between the objective and subjective quality assessments obtained using the neural network. The training part is sub-divided into normal sample and abnormal sample. These two samples are given to the mapping functions. In the testing part, the isolated points were identified and the testing images was then assessed by using the new method. Here the SVM classifier is used. This classifier is used to analyze data and recognize patterns used for classification and regression analysis.hence it takes the set of input data and predicts for each given input which of two possible classes forms the output.therefore the mapping function maps the output of normal sample with the output of SVM classifier type one,thus produces the result as ouputland maps the output of abnormal sample with the output of SVM classifier type two,thus produces the result as ouput2.

\subsection{Image quality assessment category}

There are several techniques and metrics that can be measured objectively and automatically evaluated by a computer program. Therefore, they can be classified as full-reference (FR) methods and no-reference (NR) methods.In FR image quality assessment methods, the quality of a test image is evaluated by comparing it with a reference image that is assumed to have perfect quality. NR metrics try to assess the quality of an image without any reference to the original one. For example, comparing an original image to the output of JPEG compression of that image is full-reference - it uses the original as reference.

\subsection{Image Quality Measure based on Error Sensitivity:}

\subsubsection{Mean Squared Error:}

Mean Squared Error is the most commonly used image quality measure. The goal of this measure is to compare two images by providing the quantitative score that describes the degree of similarity or the level of distortion/error between the two images.Consider an image Xij of size MXN, and another image of same size Yij $\{\mathrm{i}=1 \ldots \mathrm{M}\},\{\mathrm{j}=1 \ldots \mathrm{N}\}$ consisting of MXN pixels. The Mean Squared Error between the two image is given by Mean Squared Error(MSE).

$$
\operatorname{MSE}[f(n), g(n)]=\frac{1}{N} \sum_{n}[f(n)-g(n)]^{\wedge} 2
$$

The error image $e(n)=f(n)-g(n)$ is the difference between the original image and distorted image. If one of the image is an original image of acceptable quality, and the other is a distorted image whose quality is to be evaluated, MSE gives the measure of image quality.

\subsubsection{Peak Signal to Noise Ratio:}

In image processing, MSE is converted into Peak signal to noise ratio (PSNR) given by

$$
\operatorname{PSNR}[f(n), g(n)]=10 \log 10 E^{2} / \operatorname{MSE}[f(n), g(n)]^{2}
$$


where $\mathrm{L}$ is the dynamic range of the allowable image pixel intensities. For example, for image that have allocations of $8 \mathrm{~b} /$ pixel of gray scale, $\mathrm{L}=28-1=255$. The PSNR is useful if images having different dynamic ranges are being compared, but otherwise contains no new information.

\subsubsection{Advantages of Error Sensitivity Measurement:}

MSE, PSNR have many attractive features:

1) It is simple to calculate. It is parameter free and inexpensive to compute. It has complexity of only one multiplication and two additions per pixel.

2) It is memory less. The squared error can be evaluated at each sample, independent of other sample.

3) It has clear physical meaning. It defines the energy of the noise image. The energy is preserved even after applying linear transformation, such as Fourier Transform on the image. Hence this assures that the energy of the distortion remains same for transform domain and spatial domain.

\subsubsection{Limitations:} quality.

There are a number of reasons why MSE or PSNR may not correlate well with the human perception of

1] Digital pixel values, on which the MSE is typically computed, may not exactly represent the light stimulus entering the eye.

2] Simple error summation, like the one implemented in the MSE formulation, may be markedly different from the way the HVS and the brain arrives at an assessment of the perceived distortion.

3] Two distorted image signals with the same amount of error energy may have very different structure of errors, and hence different perceptual quality.

\begin{tabular}{|c|c|c|c|c|}
\hline & \multicolumn{4}{|c|}{ VI. Result } \\
\hline NO & $\begin{array}{l}\text { QUALITY } \\
\text { INDEX }\end{array}$ & $\begin{array}{l}\text { ORIGINAL } \\
\text { IMAGE }\end{array}$ & $\begin{array}{l}\text { TEST } \\
\text { IMAGE } \\
1\end{array}$ & $\begin{array}{l}\text { TEST } \\
\text { IMAGE } \\
2\end{array}$ \\
\hline 1 & PSNR & 0 & 0.012916 & 0.049194 \\
\hline 2 & BIAS & $\mathbf{0}$ & 22.66615 & 2.85665 \\
\hline 3 & MAD & 1 & 0.688163 & 0.310855 \\
\hline 4 & RMSE & 0 & 9.369445 & 10.7194 \\
\hline 5 & $\mathrm{CC}$ & 1 & 0.664519 & 0.12727 \\
\hline 6 & $\begin{array}{l}\text { ENTROPY } \\
\text { DIFFERENCE }\end{array}$ & $\mathbf{0}$ & 0.027394 & 1.058842 \\
\hline 7 & ERGAS & $\mathbf{0}$ & 2.286058 & 2.615434 \\
\hline 8 & RASE & 0 & 9.176059 & 11.43722 \\
\hline
\end{tabular}

\section{Software Used}

MATLAB, which stands for MATrix LABoratory, is a powerful, general-purpose system or environment for doing mathematics, scientific and engireeng calculations.MATLAB is a "High-Performance Numeric Computation and Visualization Software" package.MATLAB has a number of add-on software modules, called toolbox , that perform more specialized computations. Neural Network Toolbox ${ }^{\mathrm{TM}}$ provides functions and apps for modeling complex nonlinear systems that are not easily modeled with a closed-form equation. Neural Network Toolbox supports supervised learning with feedforward, radial basis, and dynamic networks. It also supports unsupervised learning with self-organizing maps and competitive layers. With the toolbox you can design, train, visualize, and simulate neural networks

\section{Conclusion}

The concept of isolated points was developed to better define image quality. The isolated points analysis wasused to develop a neural network and SVM based image quality assessment method using PSNR and SSIM as two image quality indexes. Tests show that the method more accurately reflects image quality and reduces the number of isolated points in the performance curve. To get more accurate assessment, more HVS characteristics should be analyzed to develop high-performance quality assessment methods.

\section{References}

[1]. ITU-R Recommendation BT. 500-10 (2000). Methodology for the subjective assessment of the quality of the television pictures. 2000.

[2]. Vapnik V N. An overview of statistical learning theory. IEEE Transactions on Neural Networks, 1999, 10(5): 988-999.

[3]. Li Guiling, Wang Nannan, Zhang Qiang. Study of moving image quality evaluation base on MPEG-2 system. Journal of Tianjin University, 2001, 34(5): 573-576. (in Chinese) 
[4]. Wolfgang Maass. Networks of spiking neurons: The third generation of neural network models. Neural Networks, 10(9):1659 \{ 1671, 1997.

[5]. Sander M. Bohte, Joost N. Kok, and Han La Poutre. Error back-propagation in temporally encoded networks of spiking neurons. Neuro Computing, 48:17-37, 2002.

[6]. A P Braga, T B Ludemir, and André C P L F de Carvalho. Artificial Neural Networks Theory and Applications (in portuguese). LTC Editora, Rio de Janeiro, 1st edition, 2000.

[7]. Sander Marcel Bohte. Spiking Neural Networks. PhD thesis, Universiteit Leiden, Mar 2003.

[8]. Frank C. Hoppensteadt and Eugene M. Izhikevich. Canonical neural models. In M.A. Arbib, editor, Brain Theory and Neural Networks. The MIT Press, 2nd. edition, 2001.

[9]. [HL03] Duane Hanselman and Bruce Littlefield. Mastering MATLAB 6: A Compreensive Tutorial and Reference (in portuguese). Prentice Hall, 2003. 